\title{
ARBEITSVERTRÄGE
}

\section{Wiederholte Befristungen auf dem Prüfstand}

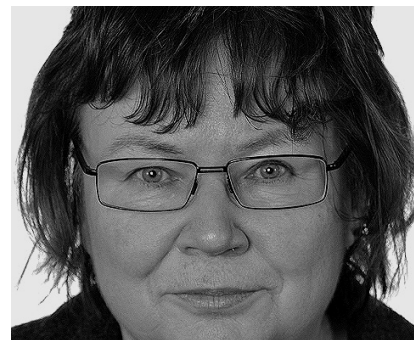

VON HELGA SPINDLER

Prof. Dr. Helga Spindler ist Hochschullehrerin für öffentliches Recht mit Schwerpunkt Sozialrecht und Arbeitsrecht an der Universität Duisburg-Essen. E-Mail helga.spindler@uniduisburg-essen.de

\author{
Kettenarbeitsverträge sind auch in sozialen Arbeitsfel- \\ dern nicht mehr unüblich. Verschiedene Gerichtsent- \\ scheidungen der letzten Zeit lassen jedoch begründete \\ Zweifel aufkommen, ob wiederholte Befristungen bei \\ öffentlichen und sozialen Dienstleistungen auf Dauer \\ mit dem Europarecht vereinbar sind.
}

Noch liegt » nur « die arbeitsrechtliche Befristungspraxis der Justizverwaltung Nordrhein-Westfalen beim Europäischen Gerichtshof vor. Doch die Bundesagentur für Arbeit und kleinere Vereine, die im öffentlichen Auftrag Schulhelferinnen einstellen, sind wegen unzulässiger Befristung beim Bundesarbeitsgericht schon unterlegen. Die Rechsprechung wird sich ausweiten und soziale Organisationen sollten ihre Praxis kritisch untersuchen.

\section{Bestehende Rechtslage}

Es gibt eine europäische Rahmenvereinbarung über befristete Arbeitsverträge im Anhang der Richtlinie 1999/70/EG des Rates vom 28.Juni 1999. Dort heißt es in $\mathbb{S}$ Nr.1: »Um Missbrauch durch aufeinanderfolgende befristete Arbeitsverträge oder -verhältnisse zu vermeiden, ergreifen die Mitgliedstaaten nach der gesetzlichen oder tariflich vorgeschriebenen ... üblichen Anhörung ... wenn keine gleichwertigen gesetzlichen Maßnahmen zur Missbrauchsverhinderung bestehen, unter Berücksichtigung der Anforderungen bestimmter Branchen und/oder Arbeitnehmerkategorien eine oder mehrere der folgenden Maßnahmen: a) sachliche Gründe, die die Verlängerung solcher Verträge oder Verhältnisse rechtfertigen b) die insgesamt maximal zulässige Dauer aufeinanderfolgender Arbeitsverträge oder -verhältnisse c) die zulässige Zeit der Verlängerung solcher Verträge oder Verhältnisse. «

Vor diesem Hintergrund ist nunmehr die Möglichkeit, eine Befristung mit einem anerkannten Sachgrund nach $\mathbb{S} 14$ Abs.1 Teilzeit- und Befristungsgesetz (TzBfG) zu vereinbaren, zunehmender Kritik ausgesetzt. Es geht um die zwei Befristungsgründe, die im öffentlichen Dienst, bei gemeinnützigen Einrichtungen und sozialen Dienstleistern auffallend häufig auftauchen (vgl. Hohendanner, Christian: Unsichere Zeiten, unsichere Verträge? IAB Kurzbericht 14/2010): Nämlich wenn der Arbeitnehmer "zur Vertretung eines andern Arbeitnehmers beschäftigt wird « und »aus Haushaltsmitteln vergütet wird, die haushaltsrechtlich für eine befristete Beschäftigung bestimmt sind und er entsprechend beschäftigt wird «( $\$ 14$ Abs.1 Ziff. 4 und Ziff.7 TzBfG).

Der letztere Sachgrund ist erst mit der Neuregelung im Jahre 2000 in das Gesetz aufgenommen worden. Damit wurde ein Befristungsgrund zusätzlich aufgenommen, der vorher nur für den Teilbereich der Hochschulen gegolten hat und die Lage für die Arbeitnehmer in allen öffentlich finanzierten Bereichen verschlechtert hat. Diese Befristungen stehen einer oder mehreren weiteren Befristungen mit jeweils neuen Gründen nicht entgegen und das kann zu jahrelangen Kettenarbeitsverträgen führen. Dies wird zunehmend als Ver- 
stoß gegen die europäische Regelung zur Missbrauchsverhinderung wahrgenommen.

Die jüngste Entscheidung des Bundesarbeitsgerichts betrifft eine Angestellte der nordrhein-westfälischen Justizverwaltung, die über zehn Jahre mit insgesamt 13 befristeten Arbeitsverträgen zur Vertretung jeweils verschiedener anderer Mitarbeiterinnen, die sich in Elternzeit oder Sonderurlaub befanden, kontinuierlich angestellt war (Az.: 7 AZR 443/09 (A)). Das Bundesarbeitsgericht sieht vor allem das Problem, ob der Sachgrund der Vertretung noch greift, wenn beim Arbeitgeber ein ständiger Vertretungsbedarf besteht, der durch Stammkräfte gedeckt werden könnte, die aus unbefristet eingestellten Arbeitskräften bestehen. Deshalb hat es das Verfahren dem Europäischen Gerichtshof vorgelegt. Ein weiterer Vorlagebeschluss kommt vom Landesarbeitsgericht Köln (Az.: 7 Sa 1224/09), das in einem ähnliche Fall die gleichstellungsrechtliche Problematik andeutet. Statistisch ist nachweisbar, dass die Vertretung wegen familiärer Verpflichtungen besonders regelmäßig in den hauptsächlich von Frauen besetzten Arbeitsfeldern auftaucht (IAB a. a. O., S. 5). Damit stellt sich zusätzlich noch die Frage der Diskriminierung wegen des Alters ( $\mathbb{1}$ AGG): Eine junge Berufsanfängerin ermöglichte 13 (vermutlich) älteren Mitarbeiterinnen die sozial abgesicherte Freistellung aus familiären Gründen, ohne die Möglichkeit zu haben, selbst eine Festanstellung mit entsprechendem Schutz für eine Familiengründung zu erreichen.

Außerdem hat das Bundesarbeitsgericht (Az.: 7/AZR 485/09(A)), dem Landesarbeitsgericht Köln (a. a. O.) folgend, einen weiteren Fall aus der Justizverwaltung Nordrhein-Westfalens vorgelegt, in dem wieder kontinuierlich beschäftigt worden war und zwar zuletzt befristetet wegen Haushaltsmitteln nach $\$ 14$ Abs.1 Ziff. 7 TzBfG, die nach dem Haushaltsplan 2006 für die Anstellung von Aushilfskräften benutzt werden konnten. Das Bundesarbeitsgericht hält hier besonders für klärungsbedürftig, ob es vertretbar ist, für den öffentlichen Dienst einen zusätzlichen Grund zur Befristung vorzusehen, der der Privatwirtschaft nicht zur Verfügung steht. Das Landesarbeitsgericht hat dazu ausgeführt: »Da Haushalts- pläne üblicherweise nur für begrenzte Zeiträume aufgestellt zu werden pflegen, könnte es mit einer solchen Begründung auch gerechtfertigt werden, im öffentlichen Dienst ausschließlich befristete Arbeitsverhältnisse abzuschließen. «

In einem weiteren Urteil (Az.: 7 AZR $843 / 08$ ) hat das Bundesarbeitsgericht bereits gegen die Bundesagentur für Arbeit entschieden, die 5.000 Kräfte von 2005 bis Dezember 2007 befristet nach \ 14 Abs.1 Ziff. 7 TzBfG eingestellt hatte. Es sieht hier Verfassungs- und Europarecht dadurch verletzt, dass die Zweckbestimmung für die Befristung keine Nachprüfung anhand objektiver Umstände ermöglicht und dass sich nicht erkennen lässt, für welche vorübergehenden Aufgaben die Mittel bereitgestellt werden. Der Haushaltplan enthielt nur pauschal die Ermächtigung für 5.000 Stellen, ohne deren Aufgaben näher zu erläutern. Die Verwaltung des SGB II ist aber eine Daueraufgabe der Bundesagentur. Allein die vage Erwartung, im Jahr 2007 könne weniger Arbeit anfallen, sei keine Begründung für den zukünftigen Beschäftigungsbedarf.

Nach einem älteren Urteil des Bundesarbeitsgerichts (Az.: 7 AZR 758/98) hat das auch Folgen für beauftragte Dienstleister. Dort ging es um circa 250 Schulhelferinnen, die bei einer gemeinnützigen $\mathrm{GmbH}$ angestellt waren, die diese wiederum dem Land Berlin zur Verfügung stellte. Die Schulhelferinnen wurden entsprechend der jährlich erfolgenden Anforderungen des Landes auch nur jährlich befristet eingestellt; die Klägerin im Verfahren schon vier Jahre hintereinander. Auch dies war unwirksam, insbesondere, weil die finanzielle Abhängigkeit von Haushaltsmitteln des Landes nicht als Sachgrund gelten könne. Dies gelte nur, wenn die Beschäftigung von vornherein für ein begrenztes Projekt geplant sei, nicht aber wenn die Maßnahme Teil einer Daueraufgabe des staatlichen Auftraggebers darstelle. Die Ungewissheit des Trägers, ob er einen Anschlussvertrag des Auftraggebers erhalte, dürfe nicht auf die Arbeitnehmer abgewälzt werden. Wären die Schulhelferinnen direkt beim Land angestellt, könnten sie auch nicht wirksam befristet werden. »Dann kann aber auch durch das Dazwischenschalten eines weitgehend vom Land Berlin abhängigen privaten
Arbeitgebers kein Sachgrund zur Befristung ... geschaffen werden. Vielmehr würde durch die Anerkennung einer solchen Konstruktion der durch die Befristungskontrolle gewährte arbeitsrechtliche Bestandsschutz umgangen. «

\section{Auswirkungen für die Sozialwirtschaft}

So richtig diese Überlegungen sind, so wenig sind sie bisher für die Sozialwirtschaft zu Ende gedacht. Besonders problematisch wird es, wenn die Befristungen auf einjährige Bewilligungen oder Vergabeverfahren zurückzuführen sind. Selbst wenn man die Ausgangsüberlegung durchhält, dass Daueraufgaben auch in Dauerarbeitsverhältnissen zu leisten sind, überwälzt die öffentliche Hand durch jährliche Vergabe oder Leistungsvereinbarungen für diese Dienstleistungen das Risiko der Daueranstellung auf von ihr abhängige soziale Arbeitgeber, die keine Möglichkeit haben, das finanzielle Risiko abzufangen und diese Leistungen am Markt anzubieten.

Das verlangt grundsätzlich veränderte Rahmenbedingungen. Vier Optionen sind denkbar: Entweder die Aufträge erstrecken sich über längere Zeiträume; oder der öffentliche Auftraggeber übernimmt sämtliche Folgekosten für betriebsbedingte Kündigungen; oder er verpflichtet mit der Auftragsvergabe den neuen Träger, die bisherigen Beschäftigten zu bestehenden Bedingungen zu übernehmen (wie bei einem Betriebsübergang); oder er stellt eine relevante Zahl von Beschäftigten wieder selbst an. Arbeitsfelder, in denen Befristungen gehäuft auftreten (z. B. Weiterbildung, Beschäftigungsmaßnahmen, Jugendhilfe) müssen grundsätzlich anders finanziert werden, um den Beschäftigten eine Berufsperspektive zu ermöglichen und sie nicht gegenüber andern Branchen strukturell schlechter zu stellen. So wie das heute organisiert ist, genügt es nicht den Anforderungen an eine verlässliche Bereitstellung öffentlicher Dienstleistungen.

Die Vorlage erster Fragen zu missbräuchlicher Befristung an den Europäischen Gerichtshof sollte genutzt werden, die Arbeitsbedingungen in den betroffenen Bereichen der Sozialwirtschaft auf den Prüfstand zu stellen. 